\title{
Éditorial
}

\section{Vers de nouvelles normes en environnement alimentaire pour le Canada}

\author{
Lana Vanderlee, Ph. D., rédactrice invitée (1); Erin P. Hobin, Ph. D., Dt. P. (2)
}

\section{Diffuser cet article sur Twitter}

Notre environnement alimentaire, constitué du cadre physique et social qui nous entoure, a en quelque sorte "normalisé » les comportements alimentaires malsains au Canada. L'omniprésence dans notre vie quotidienne d'aliments peu sains, pauvres en nutriments et à densité énergétique élevée a conduit à ce qu'ils constituent la norme de nos habitudes alimentaires. La facilité d'accès à ces aliments, leur faible prix, leur sapidité élevée et le marketing intensif dont ils font l'objet ont conduit à ce qu'ils constituent la préférence alimentaire de la population, pourtant non compatible avec les objectifs nutritionnels que l'on devrait viser, ce qui explique que l'alimentation de la grande majorité des Canadiens soit de piètre qualité ${ }^{1}$. Comment faire en sorte qu'une "saine alimentation " devienne la nouvelle norme à la fois attendue, réclamée et préférée par la population?

De nombreuses préférences alimentaires sont malléables et peuvent être façonnées au fil du temps par des influences externes ${ }^{2,3}$. Des experts ont ainsi défini un ensemble d'options stratégiques susceptibles de contribuer à normaliser et à accroître les choix alimentaires sains ${ }^{2}$. Certaines de ces options stratégiques ont déjà été examinées dans les numéros spéciaux précédents de la revue consacrés à l'environnement alimentaire au Canada, notamment les pratiques commerciales et publicitaires, les préparations alimentaires à teneur réduite en sel, en sucre et en gras ainsi que l'accès physique à davantage d'options santé et à moins d'options malsaines en matière de restauration. Les articles de notre dernier numéro spécial sur l'environnement alimentaire proposent d'autres options stratégiques liées à l'environnement alimentaire visant à modifier l'accès aux aliments et à leur disponibilité, de même que les perceptions du système alimentaire, facteurs ayant tous un rôle à jouer dans le façonnement des choix et des préférences alimentaires. Il demeure qu'il est peu probable que l'environnement alimentaire change par lui-même si la population ne cherche pas de son côté à prioriser les aliments riches en nutriments là où elle se rend. L'environnement alimentaire et les préférences alimentaires vont donc probablement prendre simultanément une direction plus saine, étant donné qu'ils se renforcent mutuellement. Augmenter la disponibilité en aliments nutritifs dans notre vie quotidienne va contribuer à « normaliser » leur présence et à réduire les obstacles aux choix santé, ce qui influencera les préférences et la demande. Plus le public sera sensibilisé et informé de l'importance de faire le choix d'une alimentation saine, plus il exigera que les aliments sains soient facilement accessibles et à prix abordable, et notre système alimentaire devra répondre à ces exigences.

Ce numéro contient un commentaire du Bureau du dentiste en chef du Canada, qui décrit l'impact de l'environnement alimentaire actuel sur la santé buccodentaire au Canada ${ }^{4}$. On y examine la normalisation des aliments et des boissons riches en sucre ajouté (en mettant l'accent sur les boissons sucrées) comme partie intégrante de l'alimentation des enfants, des jeunes et des jeunes adultes au Canada, et son impact négatif sur la santé buccodentaire. L'article explore la création d'environnements alimentaires qui rendent les choix plus sains davantage disponibles, abordables et accessibles (et par conséquent, plus 'normaux'), ce qui pourrait potentiellement réduire le fardeau de la piètre santé buccodentaire, ainsi que d'autres maladies non transmissibles. En dernier lieu, les auteurs soulignent l'importance de la contribution des experts en santé buccodentaire dans la conversation sur l'environnement alimentaire.

Modifier l'éventail des aliments offerts systématiquement aux consommateurs dans divers milieux peut grandement influencer les choix alimentaires, comme Raine et ses collègues l'ont montré5. Les milieux fréquentés par les enfants, que ce soit les écoles ou les centres de loisirs, jouent tout particulièrement un rôle essentiel dans le développement des préférences alimentaires, en façonnant les comportements alimentaires et en implantant des normes sociales et culturelles à des âges où l'on est très impressionnable. Les établissements de santé et autres établissements financés par le gouvernement servent de modèle au grand public en matière d'aliments jugés adéquats pour la clientèle, étant donné que ce sont les établissements officiellement les plus concernés par la santé de la population et les coûts des soins de santé. L'article présente les recommandations consensuelles formulées par plusieurs chefs de file du domaine à l'échelle du Canada en matière d'approvisionnement en aliments sains et d'offre d'aliments de ce type dans les établissements du secteur public, et il souligne également le rôle essentiel que doivent jouer les nombreux intervenants concernés afin que la mise en place de politiques d'approvisionnement en aliments sains à grande échelle soit un succès.

McIsaac et ses collègues proposent une réflexion sur l'implantation potentielle d'une politique d'approvisionnement à l'échelle

Rattachement des auteures :

1. Department of Nutritional Sciences, University of Toronto, Toronto (Ontario), Canada

2. Promotion de la santé, prévention des maladies chroniques et des traumatismes, Santé publique Ontario, Toronto (Ontario), Canada

Correspondance : Lana Vanderlee, Department of Nutritional Sciences, University of Toronto, 150 College St., Toronto (Ontario) M5S 3E2; tél. : 416-946-7545; courriel : lana.vanderlee@utoronto.ca 
locale, en l'occurrence dans les centres de loisirs et de sport ${ }^{6}$. Nous sommes ici en présence de normes et de valeurs sociales bien ancrées en matière d'aliments que l'on s'attend à retrouver dans les centres de loisirs et de sport au Canada, les consommateurs exigeant des produits moins bons pour la santé dans ces environnements. L'article fait également état des opinions à propos de la suggestion que les endroits publics décident des choix alimentaires du consommateur, et à propos du concept de responsabilité individuelle en ce qui a trait aux choix alimentaires liés à l'obésité. Les conclusions de cette étude font état, d'une part, des changements nécessaires dans les normes et les valeurs culturelles relatives aux demandes du consommateur et à la responsabilité individuelle en matière de choix alimentaires afin que des politiques d'approvisionnement en aliments sains soient mises en place avec succès et, d'autre part, des modalités potentielles de changement dans les attentes du consommateur susceptibles de survenir au fur et à mesure de la mise en place de ces nouvelles politiques dans les différents milieux.

Le rapport de situation effectué par Coleman et ses collègues rend compte d'une nouvelle approche en matière d'approvisionnement en aliments sains dans les organismes communautaires avec la mise en œuvre de l'initiative FoodReach, un programme qui réunit de petits organismes pour leur permettre d'augmenter leur pouvoir d'achat lorsqu'ils se procurent des produits alimentaires sains et frais ${ }^{7}$. Cette approche constitue un bon exemple de la manière de mettre en place avec succès des politiques d'approvisionnement en aliments sains pour de petits organismes. La création d'un portail d'échange de connaissances va permettre de favoriser les apprentissages réciproques en lien avec les expériences des différents programmes et politiques, ce qui constituait l'une des recommandations consensuelles d'experts citées par Raine et ses collègues. De plus, le programme FoodReach permet aux groupes communautaires de façonner des comportements alimentaires sains lors de leurs activités, que ce soit en nutrition pour les écoles et les centres de la petite enfance ou dans les centres de ressources qui viennent en aide aux sans-abris.

Dans le dernier article de ce numéro spécial, Martin et Vold étudient de manière qualitative l'incidence d'un projet d'agriculture urbaine auprès de sept jeunes adultes membres ou non des Premières
Nations qui ont pris part à ce projet en tant que stagiaires ${ }^{8}$. Le projet Askîy, mis sur pied à Saskatoon (Saskatchewan), montre la mesure de la déconnexion entre la population et le système alimentaire traditionnel, ce dernier étant souvent très éloigné des consommateurs lorsqu'ils explorent les allées de leur épicerie, leur menu au restaurant ou le contenu de leur gardemanger. Comme cet article l'expose, les projets agricoles en milieu urbain ont plusieurs raisons d'être et ont des répercussions positives sur l'environnement, la spiritualité, la société, l'agriculture et la littératie alimentaire. L'intégration des connaissances et des enseignements des Premières Nations rend ce projet particulièrement exceptionnel. Plus largement, ce projet fournit une illustration intéressant de la manière dont des programmes de ce type peuvent être intégrés dans les collectivités locales afin de renforcer la participation à la production d'aliments et au système alimentaire en général, de même que les valeurs liées aux produits locaux et à l'origine des aliments et les attitudes à l'égard d'une saine alimentation.

Alors que le Canada progresse vers la mise en œuvre de différentes composantes de la Stratégie en matière de saine alimentation ${ }^{9}$ et d'Une politique alimentaire pour le Canada $^{10}$, deux projets qui soulignent l'importance cruciale des politiques de saine alimentation afin de créer un environnement et des systèmes alimentaires qui encouragent cette voie, nous avons à prendre conscience du potentiel incroyable que peuvent avoir ces politiques pour changer les mentalités des consommateurs et leur faire adopter de nouvelles "normes " plus saines. Les articles de ce numéro insistent sur la nécessité d'instaurer davantage de politiques, d'outils, de programmes et de pratiques en soutien aux réformes du système alimentaire afin d'offrir des aliments plus sains et riches en nutriments de manière permanente dans tous les milieux au Canada. Ces articles fournissent également des exemples d'interventions locales qui encouragent les changements environnementaux pour rendre les aliments sains plus accessibles, plus disponibles et à moindre coût.

Si nous exigeons des aliments sains accessibles à tous, vendus à prix abordable et dont on fasse la promotion, nous allons contribuer à faire en sorte que les générations actuelles et à venir exigent, préfèrent et consomment des aliments favorisant la santé et le bien-être.

\section{Remerciements}

Lana Vanderlee est titulaire d'une bourse postdoctorale Banting des Instituts de recherche en santé du Canada.

\section{Références}

1. Garriguet D. La qualité de l'alimentation au Canada. Rapports sur la santé. 2009;20(3):41-52.

2. Hawkes C, Smith TG, Jewell J, et al. Smart food policies for obesity prevention. 2015. Lancet. 2015;385(9985): 2410-2421. doi: 10.1016/S0140-6736(14) 61745-1.

3. Birch LL. Development of food preferences. Annual review of nutrition. 1999;19(1):41-62.

4. Le Bureau du dentiste en chef du Canada. Commentaire - Qu'en est-il de la bouche? Relier la santé buccodentaire et l'environnement alimentaire. Promotion de la santé et prévention des maladies chroniques au Canada. 2018;38(1):4-6.

5. Raine KD, Atkey K, Lee Olstad D, et al. Approvisionnement en aliments sains et normes nutritionnelles dans les établissements publics : synthèse des données probantes et recommandations stratégiques consensuelles. Promotion de la santé et prévention des maladies chroniques au Canada. 2018; 38(1):7-20.

6. McIsaac JLD, Jarvis SL, Spencer R, Kirk SFL. Aperçu - Un changement difficile à faire accepter : constats d'une analyse qualitative de l'offre d'aliments sains dans les installations récréatives et sportives. Promotion de la santé et prévention des maladies chroniques au Canada. 2018;38(1): 21-25.

7. Coleman P, Gultig J, Emanuel B, Gee M, Orpana H. Rapport d'étape FoodReach Toronto : réduire le coût des aliments pour les organismes sociaux et les groupes communautaires. Promotion de la santé et prévention des maladies chroniques au Canada. 2018;38(1):26-32. 
8. Martin W, Vold L. Renforcer les compétences grâce à l'agriculture urbaine : rapport sur le projet Askîy. Promotion de la santé et prévention des maladies chroniques au Canada. 2018;38(1): 33-40.

9. Santé Canada. Stratégie en matière de saine alimentation [Internet]. Ottawa (Ont.) : Santé Canada; 2016. En ligne à : https://www.canada.ca/fr/sante -canada/services/publications/aliments -et-nutrition/strategie-matiere-saine -alimentation.html

10. Gouvernement du Canada. Une politique alimentaire pour le Canada. Ottawa (Ont.) : Gouvernement du Canada; 2017. En ligne à : https:// www.canada.ca/fr/campagne/politique -alimentaire.html 